\title{
Reliability of Ultrasonography in Confirming Endotracheal Tube Placement in an Emergency Setting
}

\author{
Vimal Koshy Thomas, Cherish Paul', Punchalil Chathappan Rajeev, Babu Urumese Palatty \\ Department of Emergency Medicine, 'Department of Anesthesia and Critical Care, Jubilee Mission Medical College and Research Institute, Thrissur, Kerala, India
}

\section{Abstract}

Background and Objectives: Over the past few years, ultrasonography is increasingly being used to confirm the correct placement of endotracheal tube (ETT). In our study, we aimed to compare it with the traditional clinical methods and the gold standard quantitative waveform capnography. Two primary outcomes were measured in our study. First was the sensitivity and specificity of ultrasonography against the other two methods to confirm endotracheal intubation. The second primary outcome assessed was the time taken for each method to confirm tube placement in an emergency setting. Methods: This is a single-centered, prospective cohort study conducted in an emergency department of a tertiary care hospital. We included 100 patients with indication of emergency intubation by convenient sampling. The intubation was performed as per standard hospital protocol. As part of the study protocol, ultrasonography was used to identify ETT placement simultaneously with the intubation procedure along with quantitative waveform capnography (end-tidal carbon dioxide) and clinical methods. Confirmation of tube placement and time taken for the same were noted by three separate health-care staffs. Results and Discussion: Out of the 100 intubation attempts, five (5\%) had esophageal intubations. The sensitivity and specificity of diagnosis using ultrasonography were $97.89 \%$ and $100 \%$, respectively. This was statistically comparable with the other two modalities. The time taken to confirm tube placement with ultrasonography was $8.27 \pm 1.54 \mathrm{~s}$ compared to waveform capnography and clinical methods which were $18.06 \pm 2.58$ and $20.72 \pm 3.21 \mathrm{~s}$, respectively. The time taken by ultrasonography was significantly less. Conclusions: Ultrasonography confirmed tube placement with comparable sensitivity and specificity to quantitative waveform capnography and clinical methods. But then, it yielded results considerably faster than the other two modalities.

Keywords: Capnography, emergency, intubation, ultrasonography

\section{INTRODUCTION}

Airway management skills are indispensable for an emergency physician. Unrecognized airway accidents such as esophageal intubation tend to occur more in emergency settings, ${ }^{[1]}$ where it is reported as $6 \%-16 \% .^{[2]}$

Numerous studies have compared methods used for distinguishing between endotracheal and esophageal placement of the tube. Visual confirmation during laryngoscopy, expansion of the chest wall during ventilation, auscultatory method, capnography, and chest X-ray are modalities currently used in practice. These techniques vary in their degree of accuracy. ${ }^{[3-6]}$

The Advanced Cardiac Life Support (ACLS) 2015 guidelines recommend continuous waveform capnography in addition to clinical assessment as the most reliable method of confirming and monitoring correct placement of an endotracheal

\begin{tabular}{|l|l|}
\hline \multicolumn{3}{c|}{ Access this article online } \\
\hline Quick Response Code: & Website: \\
& www.ijccm.org \\
\cline { 2 - 3 } & \\
\end{tabular}

tube (ETT) ${ }^{[7]}$ In 1989, in a study, Vaghadia et al. came to a conclusion that end-tidal carbon dioxide $\left(\mathrm{ETCO}_{2}\right)$ is most accurate for identifying esophageal intubation. ${ }^{[8]}$ Capnography has also been found to be the best method for rapid assessment of tube position. ${ }^{[2]}$ Capnography is considered as the gold standard, but it has many limitations. Waveform capnography works on the principle of detection of carbon dioxide. This is only possible when there is sufficient pulmonary blood flow. In conditions where pulmonary blood flow is compromised such as massive pulmonary embolism and cardiac arrest, capnography is not reliable. ${ }^{[9]}$

Address for correspondence: Dr. Cherish Paul, Department of Anaesthesia and Critical Care, Jubilee Mission Medical College and Research Institute, Thrissur, Kerala, India. E-mail: drcherish@gmail.com

This is an open access article distributed under the terms of the Creative Commons Attribution-NonCommercial-ShareAlike 3.0 License, which allows others to remix, tweak, and build upon the work non-commercially, as long as the author is credited and the new creations are licensed under the identical terms.

For reprints contact: reprints@medknow.com

How to cite this article: Thomas VK, Paul C, Rajeev PC, Palatty BU. Reliability of ultrasonography in confirming endotracheal tube placement in an emergency setting. Indian J Crit Care Med 2017;21:257-61. 
Capnography is freely available in operation theaters but not in many emergency departments (EDs). Ultrasound, on the contrary, is emerging in most EDs as it is used in point of care imaging for trauma as well for guided interventions. ${ }^{[10]}$ Ultrasound machine is portable, noninvasive, and the images are easily reproducible. ${ }^{[1]}$ Various studies have shown that ultrasound is a potential method to confirm proper ETT placement. ${ }^{[12-18]}$ In our study, we aim to find the effectiveness of tracheal ultrasonography to confirm ETT placement with the existing methods.

\section{Methods}

This study was initiated after obtaining clearance from the Institutional Research and Ethics Committee. One hundred consecutive patients presented to ED with indication for endotracheal intubation were recruited into the study. Patients with significant neck or lung pathologies that would affect the study methods were excluded from the study. Tracheal sonography was performed using a SonoSite M-Turbo linear probe (13-6 MHz). A Philips M-20 monitor with a mainstream $\mathrm{ETCO}_{2}$ analyzer was used for capnography.

Intubation was performed as per the standard hospital protocol which includes confirmation by quantitative waveform capnography and clinical methods looking for bilateral chest rise and 5- point auscultation. The tube was deemed as endotracheal if a typical square waveform capnography was observed along with detection of carbon dioxide of more than $4 \mathrm{mmHg}$ after five breaths.

The sonographer identified the placement of tube as tracheal or esophageal as follows:

- Tracheal intubation if only one air-mucosal (A-M) interface with reverberation artifact and posterior shadowing was observed [Figure 1]

- Esophageal intubation if two A-M interfaces posterior shadowing were noted, which is called a double tract sign [Figure 2].

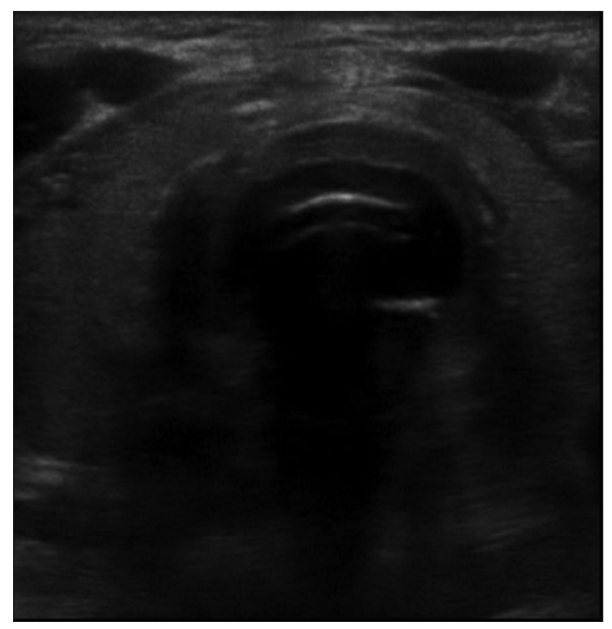

Figure 1: Sonographic image of tracheal intubation
Time zero was the time at which the person who did the intubation confirmed the completion of intubation. Following this, three different health-care staff simultaneously confirmed the tube placement, and the time taken by each method was noted using a stopwatch. End time noted was the time when individual health-care staff confirmed tube placement with their modality [Pro forma 1].

Two primary outcomes were measured in our study. First was the sensitivity and specificity of ultrasonography against the other two methods to confirm endotracheal intubation. The second primary outcome assessed was the time taken for each method to confirm tube placement in an emergency setting.

\section{RESULTS}

Among the 100 patients who underwent intubation, 59 were male and 41 were female. The mean age \pm standard deviation was $50.79 \pm 16.15$ years. The most common indication for intubation was for airway protection $(56 \%)$, followed by respiratory failure $(23 \%)$ and for hemodynamic instability (21\%).

Out of the 100 patients who underwent intubation, 95\% were tracheal and 5\% were esophageal. Tracheal ultrasonography correctly detected all 5\% of esophageal intubations but misinterpreted $2 \%$ of tracheal intubations as esophageal.

The sensitivity, specificity, positive predictive value, and negative predictive value of the ultrasound method are shown in Table 1.

The sensitivity of the ultrasonography technique was compared with that of the other two modalities using McNemar test (two tail) which showed no statistically significant difference between the groups $(P=0.47)$.

The operating time of the different modalities is shown in Table 2. Statistically ultrasonography method (T1) took significantly less time compared to clinical (T2) and waveform capnography (T3) as shown in Figure 3. This was compared

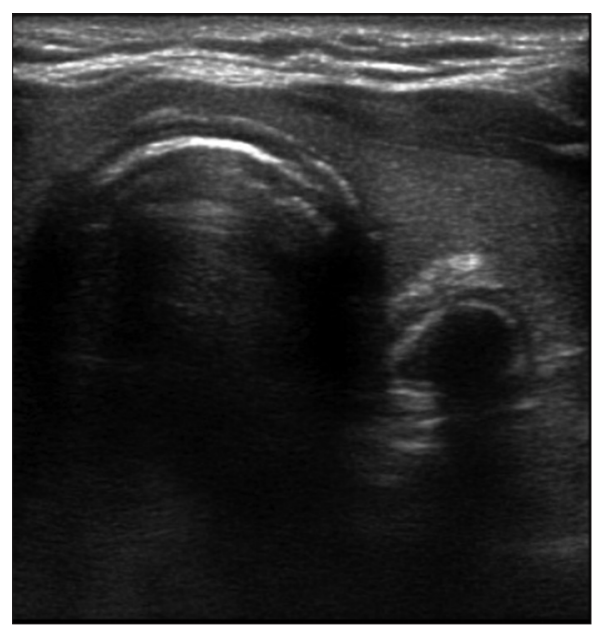

Figure 2: Sonographic image of esophageal intubation with "double tract" sign 


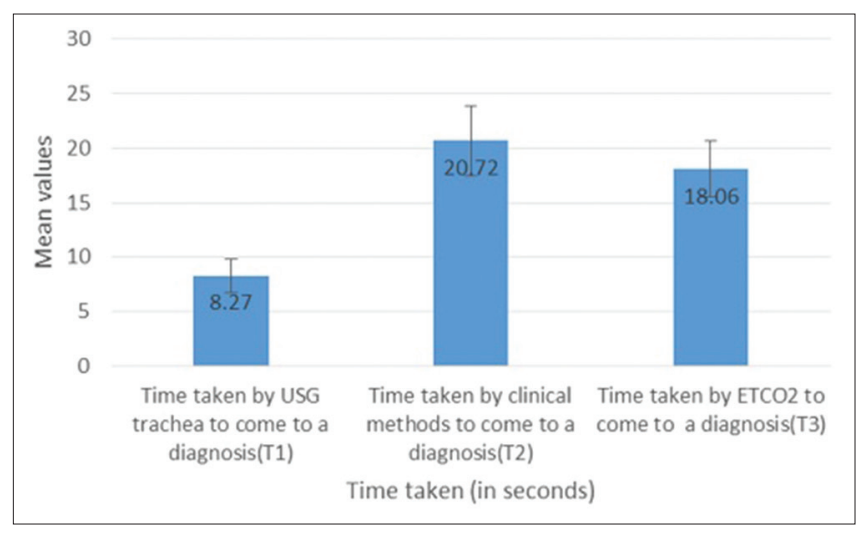

Figure 3: Time comparison

\begin{tabular}{|c|c|c|c|c|}
\hline & Sensitivity & Specificity & $\begin{array}{c}\text { Positive } \\
\text { Predictive Value }\end{array}$ & $\begin{array}{c}\text { Negative } \\
\text { Predictive Value }\end{array}$ \\
\hline $\begin{array}{l}\text { USG vs } \\
\text { Clinical }\end{array}$ & $97.89 \%$ & $100 \%$ & $100 \%$ & $71.4 \%$ \\
\hline $\begin{array}{l}\text { USG vs } \\
\text { ETCO2 }\end{array}$ & $97.89 \%$ & $100 \%$ & $100 \%$ & $71.4 \%$ \\
\hline
\end{tabular}

Table 2: Operating time of different modalities

\begin{tabular}{lcc}
\hline Time taken (in seconds) & Min-Max & Mean \pm SD \\
\hline $\begin{array}{l}\text { Time taken by USG trachea to come to a } \\
\text { diagnosis (T1) }\end{array}$ & $5.0-12.0$ & $8.27 \pm 1.54$ \\
$\begin{array}{l}\text { Time taken by clinical methods to come to a } \\
\text { diagnosis (T2) }\end{array}$ & $10.20-30.0$ & $20.72 \pm 3.21$ \\
Time taken by ETCO2 to come to a diagnosis (T3) & $13.20-28.30$ & $18.06 \pm 2.58$ \\
\hline Comparison & Difference & $\boldsymbol{P}$ \\
\hline T1- T2 & 12.46 & $<0.001^{* *}$ \\
T1- T3 & 9.79 & $<0.001^{* *}$ \\
T2-T3 & 2.66 & $<0.001^{* *}$ \\
Student $t$ test** & & \\
\hline
\end{tabular}

with Student's $t$-test $(P<0.001)$ when ultrasonography was compared with either capnography or clinical methods.

\section{DISCUSSION}

Identification of correct placement of ETT has been dependent on the airway specialist's skill in visualizing the vocal cords and on clinical methods to look for equal air entry on both lungs. ${ }^{[19]}$ The vocal cords may not always be visualized, particularly in difficult airway and emergency settings. The accuracy of any technique to identify correct ETT placement is described by its sensitivity and specificity ${ }^{[20]}$ Many methods have evolved, but no single method has proved to be $100 \%$ reliable in distinguishing between tracheal and esophageal intubations. ${ }^{[19]}$

The ACLS, 2015, mentioned the different methods for identification of ETT placement including ultrasonography by placing a transducer transversely on the anterior part of the neck above the suprasternal notch. In addition, lung sliding sign on ultrasound of the thoracic cavity can identify movement of the lung. ${ }^{[18]}$ It may also help in identifying endobronchial intubation. ${ }^{[20]}$

Quantitative waveform capnography is not widely available in EDs. ${ }^{[2]}$ In a survey of the American National Emergency Airway Registry series, a total of $77 \%$ of physicians reported that colorimetric $\mathrm{ETCO}_{2}$ detectors were available in their hospitals, but only $25 \%$ of respondents used continuous quantitative capnography. ${ }^{[22]}$ Hence, another confirmation technique with an easily available equipment is a welcome in EDs. Ultrasound is commonly used in $\mathrm{EDs}^{[10]}$ for purposes such as focused intensive care echocardiography, ${ }^{[23]}$ focused assessment of sonography in trauma, ${ }^{[24,25]}$ and for vascular access. ${ }^{[25,26]}$

Of late, ultrasound is being used in ED for the confirmation of ETT placement. ${ }^{[27]}$

The use of ultrasound to confirm ETT placement is attractive due to its portability and repeatability with good sensitivity and specificity. ${ }^{[28-31]}$ Moreover, ultrasonographic images are not affected by low pulmonary blood flow as compared to capnography ${ }^{[32]}$ Tracheal ultrasound detects esophageal intubation even before ventilating the patient, which prevents unnecessary forced ventilation to the stomach and its associated complications.

Using ultrasonography, ETT placement can be confirmed using tracheal, lung, or diaphragmatic scanning. Our study was performed using tracheal sonography which is the most common ultrasound modality used for the same. ${ }^{[12-17,28-37]}$ Transtracheal ultrasound has a sensitivity of $95.7 \%-100 \%$ and a specificity of $96.3 \%-100 \%$ in identifying ETT placement. ${ }^{[38]}$

Different authors describe different sonographic features to diagnose tracheal and esophageal intubations, but close examination of the ultrasound images revealed that almost similar features were described differently. ${ }^{[12-17,28-37]}$ To date, studies are lacking that directly compare the accuracy of different sonographic features.

Our study results had $5 \%$ of esophageal intubations which was comparable to similar studies. ${ }^{[12,29]}$

Four studies which used tracheal ultrasonography had detected $10 \%$ or more esophageal intubations ${ }^{[1,28,32,34]}$ with a high sensitivity and specificity. Three of these studies were conducted at the $\mathrm{ED}^{[15,28,32]}$ by emergency medicine (EM) residents while one was conducted at the operating room by anesthesiologists. ${ }^{[34]}$

One study which used diaphragmatic movement to confirm tube placement had $21 \%$ esophageal intubation and detected it with a lower sensitivity $(91.7 \%)$ and specificity $(95.6 \%){ }^{[15]}$

Three studies which used cadaveric models had higher esophageal intubation rates of $37 \%-50 \% \cdot{ }^{[17,31,35]}$ The sensitivity and specificity of one study was $100 \% \cdot{ }^{[17]}$ The cause for high sensitivity and specificity was probably due to the fact that the operators were qualified EM physicians and the study was conducted in a planned laboratory setting. 
The other two studies had a lower sensitivity and specificity, probably due to the fact that the operators were residents with $<12$ months' experience showing operator dependence.

The time required to confirm ETT intubation is an important consideration for any method used. Transtracheal ultrasound can be used for verification while the intubation is being performed or upon completion. Real-time sonographic imaging during intubation had higher sensitivity for detection of esophageal intubation than postintubation scanning. ${ }^{[12,15,30,32,33,35-38]}$

Using capnography, the patient's lungs would have to be ventilated 5 times for confirmation. ${ }^{[13]}$ For this reason, transtracheal ultrasound can diagnose ETT intubation faster than capnography. Various studies have reported that the time required to perform transtracheal ultrasound ranged from 5 to $45 \mathrm{~s}^{\left[{ }^{[37-39]}\right.}$ Two studies compared timeliness of ultrasound with that of capnography and found that the median verification time with ultrasound was significantly shorter than with capnography. ${ }^{[40]}$

\section{Conclusions}

Ultrasonography, end-tidal capnography, and conventional clinical methods have comparable sensitivity and specificity in identifying tracheal or esophageal position of ETT.

However, ultrasonography detected the tube placement faster than the other two methods. The time difference is statistically significant, and, considering that the scenario is time critical, it has significant clinical importance. However, multi-centric trials with larger patient groups are needed before routine use of this modality.

\section{Acknowledgment}

We are thankful to "Jubilee Mission Medical College and Research Institute" and "Kerala University of Health Sciences" for providing the resources to conduct the study.

\section{Financial support and sponsorship}

Nil.

\section{Conflicts of interest}

There are no conflicts of interest.

\section{RefERENCES}

1. Adriani J. Unrecognized esophageal placement of endotracheal tubes. South Med J 1986;79:1591-3.

2. Schwartz DE, Matthay MA, Cohen NH. Death and other complications of emergency airway management in critically ill adults. A prospective investigation of 297 tracheal intubations. Anesthesiology 1995;82:367-76.

3. Knapp S, Kofler J, Stoiser B, Thalhammer F, Burgmann H, Posch M, et al. The assessment of four different methods to verify tracheal tube placement in the critical care setting. Anesth Analg 1999;88:766-70.

4. Grmec S. Comparison of three different methods to confirm tracheal tube placement in emergency intubation. Intensive Care Med 2002;28:701-4.

5. Raphael DT, Benbassat M, Arnaudov D, Bohorquez A, Nasseri B. Validation study of two-microphone acoustic reflectometry for determination of breathing tube placement in 200 adult patients. J Am Soc Anesthesiol 2002;97:1371-7.
6. Salem MR. Verification of endotracheal tube position. Anesthesiol Clin North America 2001;19:813-39.

7. Link MS, Berkow LC, Kudenchuk PJ, Halperin HR, Hess EP, Moitra VK, et al. Part 7: Adult advanced cardiovascular life support: 2015 American Heart Association guidelines update for cardiopulmonary resuscitation and emergency cardiovascular care. Circulation 2015;132 18 Suppl 2:S444-64.

8. Vaghadia H, Jenkins LC, Ford RW. Comparison of end-tidal carbon dioxide, oxygen saturation and clinical signs for the detection of oesophageal intubation. Can J Anaesth 1989;36:560-4.

9. Cook TM, Nolan JP. Use of capnography to confirm correct tracheal intubation during cardiac arrest. Anaesthesia 2011;66:1183-4.

10. Sustic A. Role of ultrasound in the airway management of critically ill patients. Crit Care Med 2007;35 5 Suppl: S173-7.

11. Galicinao J, Bush AJ, Godambe SA. Use of bedside ultrasonography for endotracheal tube placement in pediatric patients: A feasibility study. Pediatrics 2007;120:1297-303.

12. Chou HC, Chong KM, Sim SS, Ma MH, Liu SH, Chen NC, et al. Real-time tracheal ultrasonography for confirmation of endotracheal tube placement during cardiopulmonary resuscitation. Resuscitation 2013;84:1708-12.

13. Chou HC, Tseng WP, Wang CH, Ma MH, Wang HP, Huang PC, et al. Tracheal rapid ultrasound exam (T.R.U.E.) for confirming endotracheal tube placement during emergency intubation. Resuscitation 2011;82:1279-84

14. Hsieh KS, Lee CL, Lin CC, Huang TC, Weng KP, Lu WH. Secondary confirmation of endotracheal tube position by ultrasound image. Crit Care Med 2004;32 9 Suppl:S374-7.

15. Hosseini JS, Talebian MT, Ghafari MH, Eslami V. Secondary confirmation of endotracheal tube position by diaphragm motion in right subcostal ultrasound view. Int J Crit Illn Inj Sci 2013;3:113-7.

16. Lyon M, Walton P, Bhalla V, Shiver SA. Ultrasound detection of the sliding lung sign by prehospital critical care providers. Am J Emerg Med 2012;30:485-8.

17. Weaver B, Lyon M, Blaivas M. Confirmation of endotracheal tube placement after intubation using the ultrasound sliding lung sign. Acad Emerg Med 2006;13:239-44.

18. Sim SS, Lien WC, Chou HC, Chong KM, Liu SH, Wang CH, et al. Ultrasonographic lung sliding sign in confirming proper endotracheal intubation during emergency intubation. Resuscitation 2012;83:307-12.

19. Williamson JA, Webb RK, Szekely S, Gillies ER, Dreosti AV. The Australian Incident Monitoring Study. Difficult intubation: An analysis of 2000 incident reports. Anaesth Intensive Care 1993;21:602-7.

20. Blaivas M, Tsung JW. Point-of-care sonographic detection of left endobronchial main stem intubation and obstruction versus endotracheal intubation. J Ultrasound Med 2008;27:785-9.

21. Deiorio NM. Continuous end-tidal carbon dioxide monitoring for confirmation of endotracheal tube placement is neither widely available nor consistently applied by emergency physicians. Emerg Med J 2005;22:490-3.

22. Wayne MA, Slovis CM, Pirrallo RG. Management of difficult airways in the field. Prehosp Emerg Care 1999;3:290-6.

23. Price S, Via G, Sloth E, Guarracino F, Breitkreutz R, Catena E, et al. Echocardiography practice, training and accreditation in the intensive care: Document for the World Interactive Network Focused on Critical Ultrasound (WINFOCUS). Cardiovasc Ultrasound 2008;6:49.

24. Brenchley J, Walker A, Sloan JP, Hassan TB, Venables H. Evaluation of focussed assessment with sonography in trauma (FAST) by UK emergency physicians. Emerg Med J 2006;23:446-8.

25. Maecken T, Grau T. Ultrasound imaging in vascular access. Crit Care Med 2007;35 5 Suppl:S178-85.

26. Arntfield RT, Millington SJ. Point of care cardiac ultrasound applications in the emergency department and Intensive Care Unit - A review. Curr Cardiol Rev 2012;8:98-108.

27. Moore CL, Copel JA. Point-of-care ultrasonography. N Engl J Med 2011:364:749-57.

28. Abbasi S, Farsi D, Zare MA, Hajimohammadi M, Rezai M, Hafezimoghadam P. Direct ultrasound methods: A confirmatory technique for proper endotracheal intubation in the emergency department. Eur J Emerg Med 2015;22:10-6. 
29. Adi O, Chuan TW, Rishya M. A feasibility study on bedside upper airway ultrasonography compared to waveform capnography for verifying endotracheal tube location after intubation. Crit Ultrasound J 2013;5:7.

30. Muslu B, Sert H, Kaya A, Demircioglu RI, Gözdemir M, Usta B, et al. Use of sonography for rapid identification of esophageal and tracheal intubations in adult patients. J Ultrasound Med 2011;30:671-6.

31. Göksu E, Sayraç V, Oktay C, Kartal M, Akcimen M. How stylet use can effect confirmation of endotracheal tube position using ultrasound. Am J Emerg Med 2010;28:32-6.

32. Park SC, Ryu JH, Yeom SR, Jeong JW, Cho SJ. Confirmation of endotracheal intubation by combined ultrasonographic methods in the emergency department. Emerg Med Australas 2009;21:293-7.

33. Werner SL, Smith CE, Goldstein JR, Jones RA, Cydulka RK. Pilot study to evaluate the accuracy of ultrasonography in confirming endotracheal tube placement. Ann Emerg Med 2007;49:75-80.

34. Milling TJ, Jones M, Khan T, Tad-y D, Melniker LA, Bove J, et al. Transtracheal 2-d ultrasound for identification of esophageal intubation. J Emerg Med 2007;32:409-14.

35. Ma G, Davis DP, Schmitt J, Vilke GM, Chan TC, Hayden SR. The sensitivity and specificity of transcricothyroid ultrasonography to confirm endotracheal tube placement in a cadaver model. J Emerg Med 2007;32:405-7.

36. Das SK, Choupoo NS, Haldar R, Lahkar A. Transtracheal ultrasound for verification of endotracheal tube placement: A systematic review and meta-analysis. Can J Anaesth 2015;62:413-23.

37. Hoffmann B, Gullett JP, Hill HF, Fuller D, Westergaard MC, Hosek WT, et al. Bedside ultrasound of the neck confirms endotracheal tube position in emergency intubations. Ultraschall Med 2014;35:451-8.

38. Sun JT, Chou HC, Sim SS, Chong KM, Ma MHM, Wang HP, et al. Ultrasonography for proper endotracheal tube placement confirmation in out-of-hospital cardiac arrest patients: Two-center experience. J Med Ultrasound 2014;22:83-7.

39. Saglam C, Unlüer EE, Karagöz A. Confirmation of endotracheal tube position during resuscitation by bedside ultrasonography. Am J Emerg Med 2013;31:248-50

40. Pfeiffer P, Rudolph SS, Børglum J, Isbye DL. Temporal comparison of ultrasound vs. auscultation and capnography in verification of endotracheal tube placement. Acta Anaesthesiol Scand 2011;55:1190-5.

\section{Pro Forma}

\section{Pro forma 1: Ultrasonography for Confirming Endotracheal Tube Placement}

\section{Hospital Register No:}

Age/Sex:

Vitals:

Indication:

Observer 1: Ultrasonography

Tube placement in - Encircle one

Trachea Esophagus

Total time taken by ultrasonography: T1 (in seconds)

Tube position: Encircle one

Endobronchial Tracheal

Observer 2: 5-point auscultation

Tube placement in - Encircle one

Trachea Esophagus

Time taken by clinical methods: T2 (in seconds)

Tube position: Encircle one

Endobronchial Tracheal

Observer 3: End-tidal capnography

Tube placement in: Encircle one

Trachea Esophagus

Time taken by capnography: T3 (in seconds)

Chest radiography finding

Tube position: Encircle one

Right main bronchus Central Left main bronchus Tube dislodged 